Article

\title{
The Ongoing Growth of the M87 Halo through Accretion Events
}

\author{
Alessia Longobardi ${ }^{1, *}$, Magda Arnaboldi ${ }^{2, \dagger}$ and Ortwin Gerhard ${ }^{1, \dagger}$ \\ ${ }^{1}$ Max-Planck-Institut fuer extraterrestrische Physik, Giessenbachstr.1, 85748 Garching, Germany; \\ E-Mail: gerhard@mpe.mpg.de \\ ${ }^{2}$ ESO, Karl-Schwarzschild-Str.2, 85748 Garching, Germany; E-Mail: marnabol@eso.org \\ ${ }^{\dagger}$ These authors contributed equally to this work. \\ * Author to whom correspondence should be addressed; E-Mail: alongobardi@mpe.mpg.de; \\ Tel.: +49-89-300-003-022.
}

Academic Editors: José Alfonso López Aguerri, Enrichetta Iodice and Alexei Moiseev

Received: 14 October 2015 / Accepted: 04 December 2015 / Published: 18 December 2015

\begin{abstract}
Planetary nebulas (PNs) offer a unique tool to investigate the outer regions of massive galaxies because their strong [OIII] $\lambda 5007 \AA$ emission line makes them detectable out to several effective radii from the galaxy's centre. We use a deep and extended spectroscopic survey of PNs ( 300 objects) to study the spatial distribution, the kinematics and the stellar populations in the extended outer halo of the bright elliptical galaxy M87 (NGC 4486) in the Virgo cluster. We show that in the Virgo core, M87 stellar halo and the intracluster light are two distinct dynamical components, with different velocity distributions. Moreover the synergy of the PN kinematical information and the deep V/B-band photometry revealed an ongoing accretion event in the outer regions of M87. This satellite accretion represents a non-negligible perturbation of the halo properties: beyond $60 \mathrm{kpc}$ the M87 halo is still growing with $60 \%$ of its light being added by the accretion event at the distance where it is detected.
\end{abstract}

Keywords: clusters: individual: Virgo; galaxies: halos; galaxies: individual: M 87 (NGC 4486); planetary nebulae: general 


\section{Introduction}

In the hierarchical formation of structures, mergers are believed to play a dominant role in the galaxy assembly [1] . A two-phase formation scenario [2] predicts that during the first dissipative phase (high redshift, $z \geq 2$ ) stars form quickly and build the compact innermost regions of massive galaxies (with stars rich in $\alpha$-elements; [3]); later on, minor merger events dominate the mass assembly in the outermost regions [4] and the accretion of metal-poor stars from smaller stellar systems results in the variation of the galaxy stellar properties. In galaxy clusters, where accretion and merger events are believed to be responsible for the origin of most of the stars in the outer halos and intracluster light (ICL) [5,6], an open question is whether there are any physical difference between these two components. In this framework the central galaxies' outer regions contain an enormous wealth of information. They represent the region where the transition between galaxy halo and ICL can be traced. Moreover, as dynamical scales are longer, they preserve fossil records of accretion events imprinted in the star formation history and chemical enrichment, and revealed in the form of spatially extended low surface-brightness features [7,8]. These substructures are not phase-mixed in the host galaxy potential and, therefore, can also be traced as kinematic features in the velocity phase-space [9].

The most significant challenge to investigate galaxy halos far away from their centres is the very low surface brightness of the continuum light ( $<1 \%$ of the sky value), that makes it difficult for the absorption line techniques to be successful. PNs are Post AGB-phase stars in the mass range between 1 and $8 \mathrm{M} \odot$, before they end their lives as white dwarfs. They offer a unique tool to investigate these environments owing to their strong [OIII] $\lambda 5007 \AA$ emission line, whereby $\sim 15 \%$ of the UV-luminosity of the central star is re-emitted [10] in the optical line. PN surveys in nearby clusters out to $50-100 \mathrm{Mpc}[11,12]$ showed that these single stars can be detected out to several hundreds kpc away from the galaxy's centre. They provide information on the stellar populations via the PN specific frequency, or $\alpha$-parameter, and the shape of the PN luminosity function. As a consequence of their strong [OIII] $\lambda 5007 \AA$ emission line, their line-of-sight (LOS) velocity can be measured and can be efficiently used as kinematical tracers.

At the highest density peak of the Virgo cluster, the nearest large scale structure in the Universe, lies M87 (NGC4486). Its extended cD envelope [13] together with a blue (B-V) colour gradient towards its outskirts [14] are consistent with a late build up of its stellar halo. Its close proximity ( 15 Mpc) has made it possible to measure the line-of-sight velocities (LOSVs) of hundreds of PNs [15], allowing us to pursue a detailed kinematic study of M87 beyond its brightest regions out to $150 \mathrm{kpc}$. We present this study in what follows.

\section{PN Photometric and Spectroscopic Surveys}

We first carried out an imaging survey with Suprime-Cam@Subaru that covered a $0.43 \mathrm{deg}^{2}$ region centred on M87 [16], obtaining images with a (on-band) narrow-band filter centred on the redshifted $[\mathrm{OIII}] \lambda 5007 \AA$ emission line (at the Virgo cluster distance) and a (off-band) broadband V-filter. As consequence of their bright [OIII] line, extragalactic PNs can be identified via the on-off band technique on the basis of automatic selection criteria defined around their narrow-band colours and their two-dimensional light distribution [16]. This selection led to a large and deep photometric sample of PNs, that we spectroscopically followed-up with the FLAMES@VLT. The spectra of the 287 sources 
classified as PNs are identified on the basis of their narrow and symmetric redshifted $\lambda 5007 \AA$ [OIII] emission line, the presence of the second $\lambda 4959 \AA$ [OIII] emission line, and the absence of a significant continuum. This PN sample covers the galaxy halo out to a distance of $150 \mathrm{kpc}$ in radius, and reaches 2.5 magnitude (i.e., $m_{5007}=28.7$ ) fainter than the bright-cut of the PN luminosity function. Hence, this sample is the largest, deepest and most extended one available in the outer region of M87. The layout of the FLAMES pointings on the sky, together with the coverage of the photometric Suprime-Cam survey, and PN saple is shown in Figure 1.
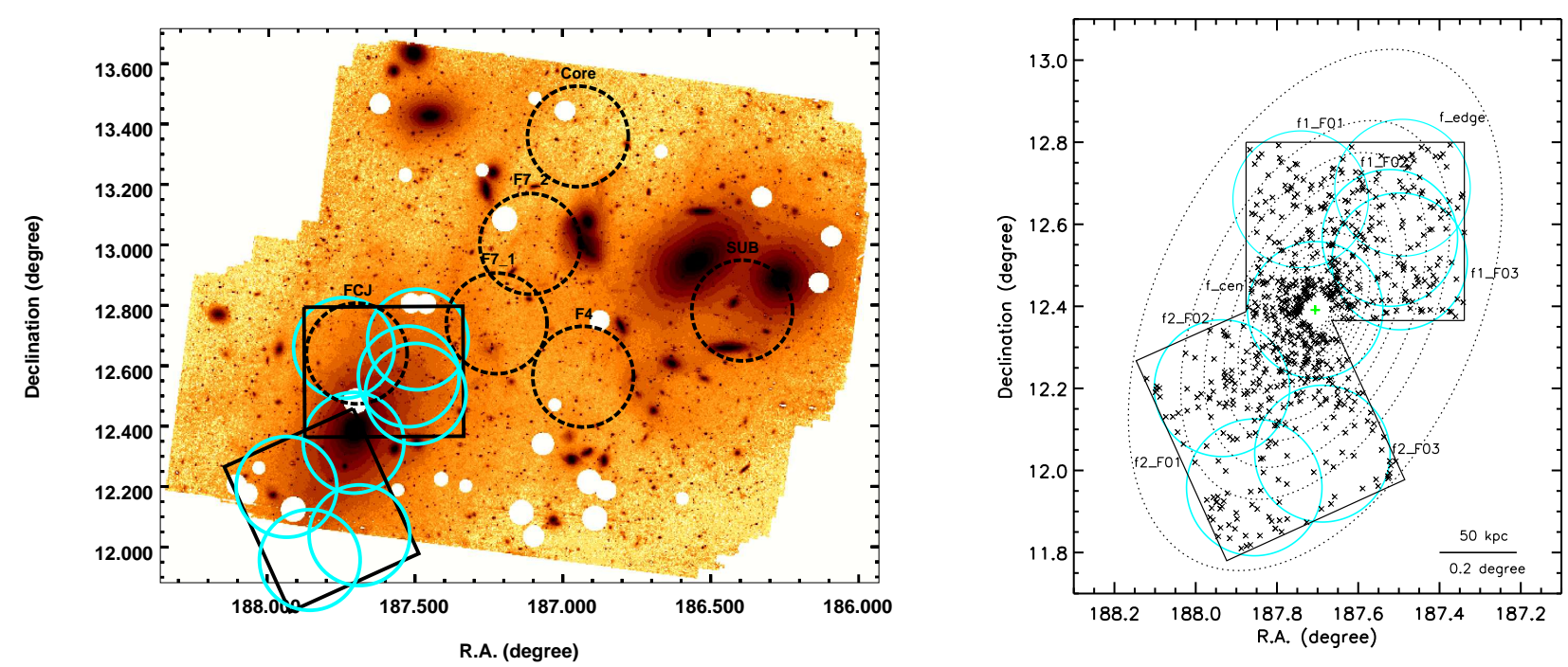

Figure 1. (Left): Image of the core of the Virgo cluster [7] with the positions of the FLAMES fields studied in this work (cyan circles) and in previous surveys (dotted black circles, see [18]). Black squares represent the Suprime-Cam survey fields used for the extraction of the photometric planetary nebulas (PN) candidates [16]; (Right): Enlarged view of the M87 halo (black rectangles). FLAMES pointings (cyan circles) and PN candidates from the photometric survey (black asterisks; [16]) are plotted over the isophotes of the M87 halo light [13]. The green cross depicts M87's centre. North is up, East is to the left.

\section{The Virgo Cluster Core: Kinematic Separation between the M87 Stellar Halo and the ICL}

At a distance of $\sim 150 \mathrm{kpc}$ from the centre of M87, it is a region at which the light of M87 blends into the ICL [17]. Previous PN studies showed that in fact in the Virgo core the ICL overlaps with the M87 stellar halo for distances larger than $60 \mathrm{kpc}$ up to $150 \mathrm{kpc}[16,18]$. This finding is further supported by the PN kinematics of our extended sample that showed the coexistence of two dynamically distinct components. The total LOSV distribution (LOSVD) has strong asymmetric wings (see Figure 2) that deviate from those of a single Gaussian distribution that is usually measured for early type galaxies [19,20]. We showed that the total LOSVD can be fitted by two Gaussian components, one related to the galaxy halo with $\mathrm{V}_{\text {halo }}=1275 \mathrm{kms}^{-1}$ and $\sigma_{\text {halo }} \simeq 300 \mathrm{kms}^{-1}$, and a broader component associated with the cluster as a whole with $\mathrm{V}_{\mathrm{ICL}}=1000 \mathrm{kms}^{-1}$ and $\sigma_{\mathrm{ICL}} \simeq 900 \mathrm{kms}^{-1}$. We then measured the M87 velocity dispersion by using a robust technique applied to the PN distribution in the projected phase-space (see Figure 2), and identify the PNs in the ICL by means of a sigma-clipping 
algorithm. The robust separation of the two PN populations led to the identification of 243 PNs for the M87 halo and 44 PNs for the ICL [15].
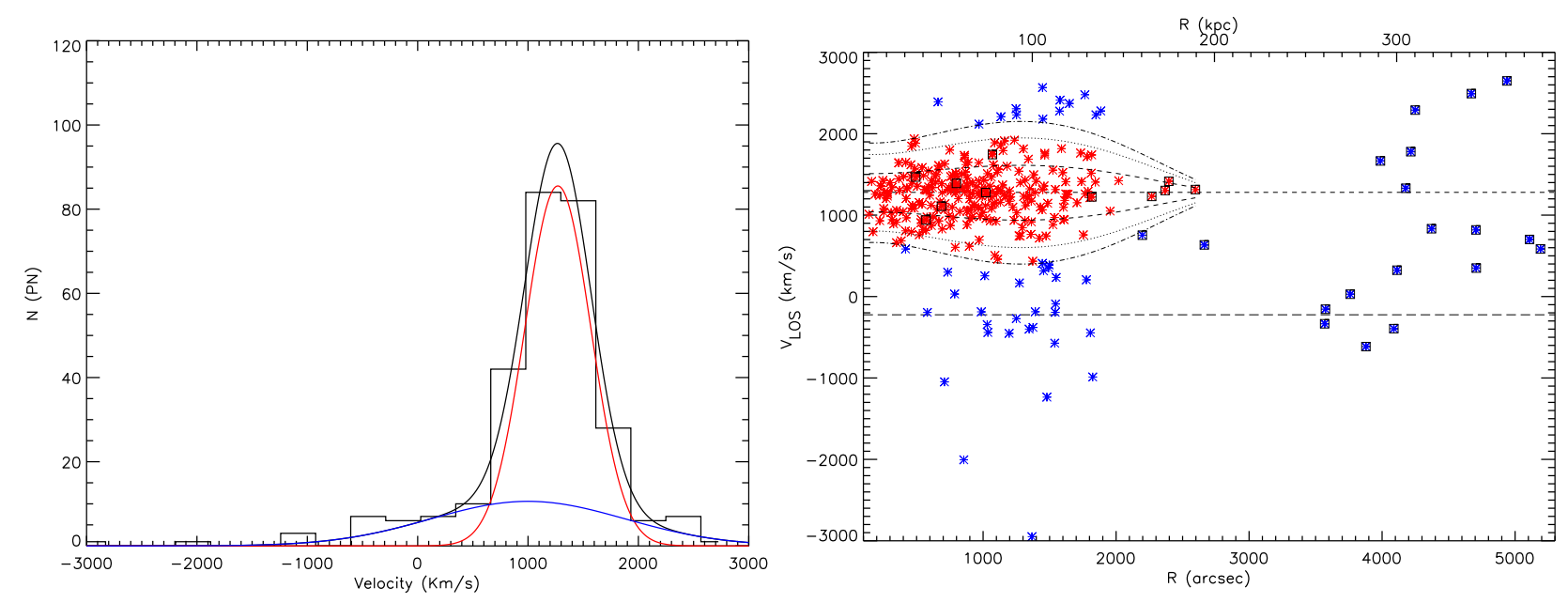

Figure 2. (Left): Line-of-sight velocities distribution (LOSVD) of the spectroscopically confirmed PNs, fit with a double Gaussian (black line). The M87 halo and intracluster light (ICL) Gaussian components are shown by the red and blue lines, respectively; (Right): LOSV plotted against major axis distance from the centre of M87 for all of the spectroscopically confirmed PNs from [15] (stars) and [18] (black-squares). The M87 halo PNs bound are denoted in red, and the ICPNs are shown in blue. The smoothed 1, 2 and 2.5 sigma thresholds are represented by the dashed, dotted and dot-dashed lines, respectively. The dashed, and long dashed lines denote M87 and M86 systemic velocities, respectively.

\subsection{The Ongoing Mass Assembly of the M87 Halo}

We discuss now the properties of the 243 halo PNs that cover a range in radii from $\sim 15$ to $150 \mathrm{kpc}$. We address the question whether the $\mathrm{cD}$ halo of M87 can be considered a smooth dynamical component or whether it shows signatures of accretion events [21]. Halos that are still forming are expected to have a complex phase-space, with accretion events signalled by the presence of colder substructures, i.e., regions in the phase-space characterised by lower velocity dispersion than the main halo (e.g., [9]) . For this purpose we implemented a Gaussian Mixture Model (GMM) algorithm on the PN phase-space, and assumed that, in elliptical annuli, the halo plus substructure LOSVDs can be described as a linear combination of $K$ independent Gaussian probability density functions (PDFs). The GMM implemented the expectation maximisation (EM) algorithm, which is an iterative process that continuously updates the PDF parameters until convergence is reached. At the end of the EM procedure, the posterior probability for a data value to belong to either the $K$ Gaussian PDFs is returned. The GMM identified 54 PNs as part of a "hidden" structure that appears as a chevron (V-shaped feature) in the phase space. To this substructure in the phase-space corresponds a substructure in the light. At the distance where the edge of the chevron is measured ( $~ 90 \mathrm{kpc}$ along the major axis and $\mathrm{V}_{\text {LOS,edge }}=1254 \mathrm{kms}^{-1}$ ), deep V-band photometry shows the presence of a crown-shaped, low-surface brightness feature. The M87 crown causes an increase in surface brightness by $\geq 60 \%$ at the location where it is detected. The crown represents the densest region of the entire substructure that extends $\sim 50 \mathrm{kpc}$ along the major axis, 
causing modifications of the ellipticity and colour profiles. From the total number of PNs, luminosity, and (B-V) colour associated to the entire accretion event, the progenitor is consistent with a galaxy of total luminosity $\mathrm{L}=2.8 \pm 1.0 \times 10^{9} \mathrm{~L}_{\odot, \mathrm{V}}$, a colour of $(\mathrm{B}-\mathrm{V})=0.75 \pm 0.05$, and a stellar mass of $\mathrm{M}=6.4 \pm 2.3 \times 10^{9} \mathrm{M}_{\odot}$ (see Longobardi et al. [21] for more details). Figure 3 shows the accretion event as kinematic substructure in the PN phase-space (top-left), as a substructure in the light and as an azimuthal variation of the colour map of the outer halo. From the distribution and velocities of the chevron PNs in Figure 3 the satellite was first disrupted entering M87 from the South (along the green dots), with the debris then moving up North, turning around in the crown region (at major axis distance $90 \mathrm{kpc}$ ), and coming back down (along magenta diamonds). The dynamical time for such an orbit is of order 1 Gyr.

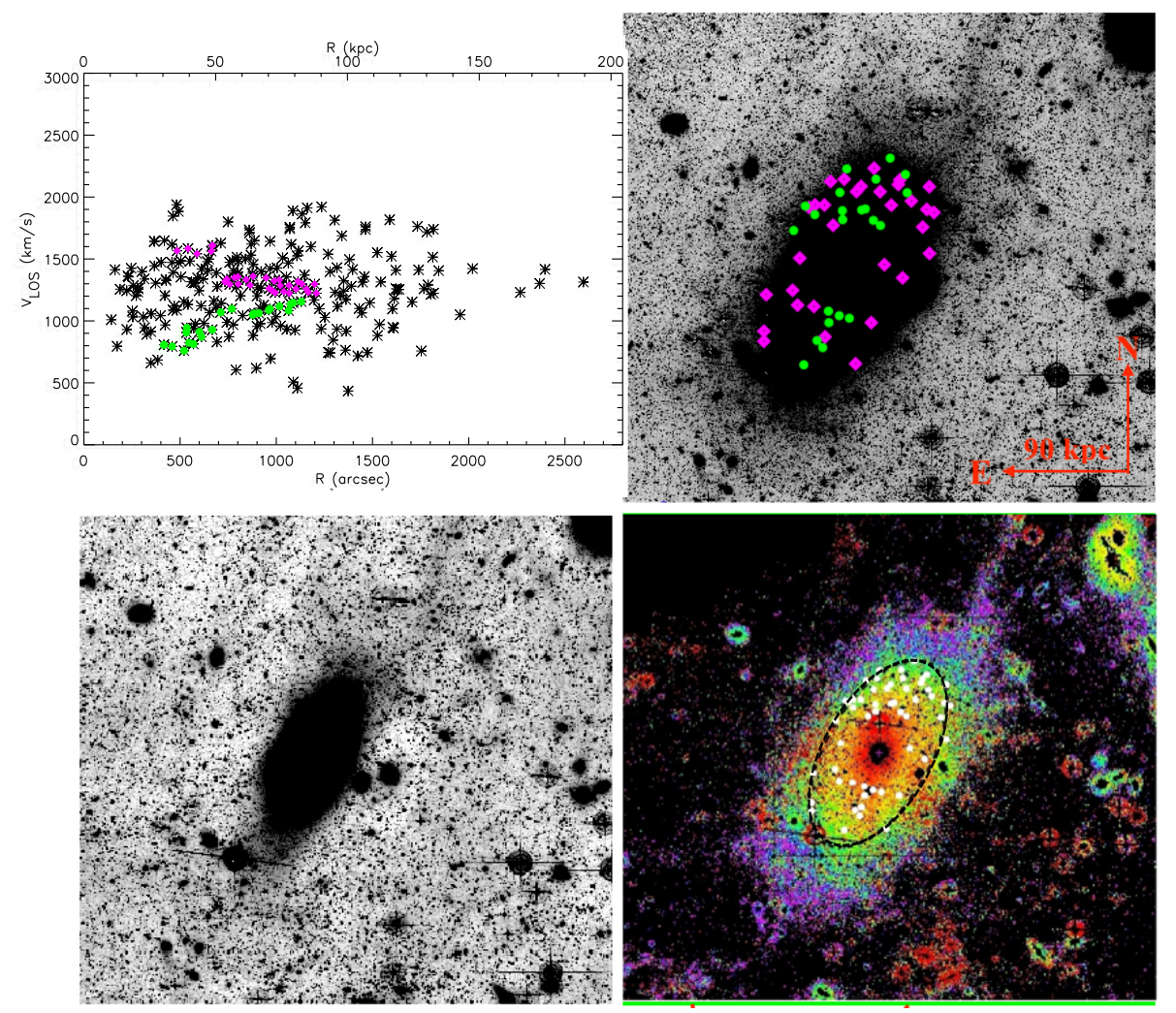

Figure 3. (Top-Left): Projected phase-space, $\mathrm{V}_{\mathrm{LOS}}$ vs. major axis distance $(R)$, for all spectroscopically confirmed PNs (black asterisks) in the halo of M87. Green circles and magenta diamonds show the PNs associated with the cold secondary peaks in the LOSVD as identified by the GMM; (Top-Right): V-band image of a $1.6 \times 1.6 \mathrm{deg}^{2}$ centred on M87 from Mihos et al. [22]. Full green circles and magenta diamonds indicate the spatial position of the M87 halo PNs in the chevron substructure, above and below $\mathrm{V}_{\mathrm{LOS} \text {,edge }}=1254 \mathrm{kms}^{-1}$, which is the LOSV at the edge of the chevron; (Bottom-Left): Unsharped masked image of M87 median binned to enhance faint structures. The crown-shaped substructure is visible at distance of $\sim 60-90 \mathrm{kpc}$ along the major axis, NW of M87; (Bottom-Right): (B-V) colour image of M87 from Mihos et al. [22] with chevron PNs overplotted (white dots). The dashed ellipse indicates the isophote at a major axis distance of $90 \mathrm{kpc}$. The crown is found in a region where the $(\mathrm{B}-\mathrm{V})$ colour is on average 0.8 , that is bluer then the average red colour on the minor axis, at the same radii. 


\section{Results and Discussion}

Using PNs as tracers we showed the stellar halo of the central galaxy M87 is distinct from the surrounding ICL: the PN velocity distribution is bimodal, where one distribution is narrow and clustered around the M87's systemic velocity and the second is broader and traces the cluster's potential. This finding is in agreement with the results from cosmological analysis of structure formation [23,24] that predict different LOSVDs for the central galaxy halo and the ICL. The different dynamics of the two components allowed for a robust identification of the M87 halo that could be further studied. Implementing GMM algorithms on the kinematics of the halo PNs we identified a chevron (V-shaped structure) as the result of a not complete phase-mixing of a disrupted satellite. From theoretical simulations, e.g., [9] in fact the chevron describes the orbits of an accretion event, and its edge identifies the distance where the stars from the satellite come to the end of their radial excursion and slow to a halt, before they reverse their motion. As a consequence of the wrapping of the satellite orbits, an enhancement of the stellar density is expected in the form of spatially extended low-surface brightness feature. For the first time such a theoretical expectation has been observationally confirmed. At the distance where we measure the edge of the chevron, deep V/B band photometry shows the presence of a spatially extended low-surface brightness feature, the crown. This accretion event has caused an important modification of the outer halo of M87: beyond $\sim 60 \mathrm{kpc}$ the halo of the central galaxy of the Virgo cluster is still assembling through the accretion of smaller systems.

\section{Acknowledgments}

Alessia Longobardi thanks the Scientific Organizing Committee (SOC) and Local Organizing Committee (LOC) for the opportunity to present these results as oral contribution.

\section{Author Contributions}

All the authors equally contributed to the reported work. All authors have read and approved the final version.

\section{Conflicts of Interest}

The authors declare no conflict of interest.

\section{References and Notes}

1. De Lucia, G.; Blaizot, J. The hierarchical formation of the brightest cluster galaxies. Mon. Not. R. Astron. Soc. 2007, 375, 2-14.

2. Naab, T.; Johansson, P.H.; Ostriker, J.P. Minor Mergers and the Size Evolution of Elliptical Galaxies. Astrophys. J. 2009, 699, L178-L182.

3. Thomas, D.; Maraston, C.; Bender, R.; Mendes de Oliveira, C. The Epochs of Early-Type Galaxy Formation as a Function of Environment. Astrophys. J. 2005, 621, 673-694.

4. Oser, L.; Ostriker, J.P.; Naab, T.; Johansson, P.H.; Burkert, A. The Two Phases of Galaxy Formation. Astrophys. J. 2010, 725, 2312-2323. 
5. Laporte, C.F.P.; White, S.D.M.; Naab, T.; Gao, L. The growth in size and mass of cluster galaxies since $\mathrm{z}=2$. Mon. Not. R. Astron. Soc. 2013, 435, 901-909.

6. Cooper, A.P.; Gao, L.; Guo, Q.; Frenk, C.S.; Jenkins, A.; Springel, V.; White, S. Surface photometry of brightest cluster galaxies and intracluster stars in $\Lambda$ CDM. Mon. Not. R. Astron. Soc. 2015, 451, 2703-2722.

7. Mihos, J.C.; Harding, P.; Feldmeier, J.; Morrison, H. Diffuse Light in the Virgo Cluster. Astrophys. J. 2005, 631, L41-L44.

8. Duc, P.-A.; Cuillandre, J.-C.; Karabal, E.; Cappellari, M.; Alatalo, K.; Blitz, L.; Bournaud, F.; Bureau, M.; Crocker, A.F.; Davies, R.L.; et al. The ATLAS3D project - XXIX. The new look of early-type galaxies and surrounding fields disclosed by extremely deep optical images. Mon. Not. R. Astron. Soc. 2015, 446, 120-143.

9. Bullock, J.S.; Johnston, K.V. Tracing Galaxy Formation with Stellar Halos. I. Methods. Astrophys. J. 2005, 635, 931-949.

10. Dopita, M.A.; Jacoby, G.H.; Vassiliadis, E. A Theoretical Calibration of the Planetary Nebular Cosmic Distance Scale. Astrophys. J. 1992, 389, 27-38.

11. Ventimiglia, G.; Arnaboldi, M.; Gerhard, O. The unmixed kinematics and origins of diffuse stellar light in the core of the Hydra I cluster (Abell 1060). Astron. Astrophys. 2011, 528, A24

12. Gerhard, O.; Arnaboldi, M.; Freeman, K.C.; Kashikawa, N.; Okamura, S.; Yasuda, N. Detection of Intracluster Planetary Nebulae in the Coma Cluster. Astrophys. J. 2005, 621, L93-L96.

13. Kormendy, J.; Fisher, D.B.; Cornell, M.E.; Bender, R. Structure and Formation of Elliptical and Spheroidal Galaxies. Astrophys. J. 2009, 182, 216-309.

14. Rudick, C.S.; Mihos, J.C.; Harding, P.; Feldmeier, J.J.; Janowiecki, S.; Morrison, H.L. Optical Colors of Intracluster Light in the Virgo Cluster Core. Astrophys. J. 2010, 720, 569-580.

15. Longobardi, A.; Arnaboldi, M.; Gerhard, O.; Hanuschik, R. The outer regions of the giant Virgo galaxy M87 Kinematic separation of stellar halo and intracluster light. Astron. Astrophys. 2015, 579, A135.

16. Longobardi, A.; Arnaboldi, M.; Gerhard, O.; Hanuschik, R. The planetary nebula population in the halo of M 87. Astron. Astrophys. 2013, 558, A42

17. Castro-Rodriguéz, N.; Arnaboldi, M.; Aguerri, J.A.L.; Gerhard, O.; Okamura, S.; Yasuda, N.; Freeman, K.C. Intracluster light in the Virgo cluster: Large scale distribution. Astron. Astrophys. 2009, 507, 621-634.

18. Doherty, M.; Arnaboldi, M.; Das, P.; Gerhard, O.; Aguerri, J.A.L.; Ciardullo, R.; Feldmeier, J.J.; Freeman, K.C.; Jacoby, G.H.; Murante, G. The edge of the M 87 halo and the kinematics of the diffuse light in the Virgo cluster core. Astron. Astrophys. 2009, 502, 771-786.

19. Gerhard, O.E. Line-of-sight velocity profiles in spherical galaxies: Breaking the degeneracy between anisotropy and mass. Mon. Not. R. Astron. Soc. 1993, 265, 213-230.

20. Bender, R.; Saglia, R.P.; Gerhard, O.E. Line-of-sight velocity distributions of elliptical galaxies. Mon. Not. R. Astron. Soc. 1994, 269, 785-813.

21. Longobardi, A.; Arnaboldi, M.; Gerhard, O.; Mihos, J.C. The build-up of the cD halo of M 87: Evidence for accretion in the last Gyr. Astron. Astrophys. 2015, 579, L3.

22. This figure is from the paper by Mihos et al., which is still in preparation. 
23. Dolag, K.; Murante, G.; Borgani, S. Dynamical difference between the $\mathrm{cD}$ galaxy and the diffuse, stellar component in simulated galaxy clusters. Mon. Not. R. Astron. Soc. 2010, 405, 1544-1559.

24. Cui, W.; Murante, G.; Monaco, P.; Borgani, S.; Granato, G.L.; Killedar, M.; de Lucia, G.; Presotto, V.; Dolag, K. Characterizing diffused stellar light in simulated galaxy clusters. Mon. Not. R. Astron. Soc. 2014, 437, 816-830.

(c) 2015 by the authors; licensee MDPI, Basel, Switzerland. This article is an open access article distributed under the terms and conditions of the Creative Commons Attribution license (http://creativecommons.org/licenses/by/4.0/). 